\title{
Discrete Power-Stepping Pulse Amplitude Modulation for Optical Camera Communications Employing a CMOS-Integrated GaN $\mu$ LED Array
}

\author{
N. Bani Hassan ${ }^{1, *}$, M. J. Strain ${ }^{1}$, M. D. Dawson ${ }^{1}$, J. Herrnsdorf ${ }^{1}$ \\ ${ }^{1}$ Institute of Photonics, Department of Physics, University of Strathclyde, Glasgow G1 1RD, UK \\ *email: navid.bani-hassan@strath.ac.uk
}

\begin{abstract}
We present discrete power-stepping $\boldsymbol{m}$-ary pulse amplitude modulation for non-line-of-sight rolling-shutter-based optical camera communications. A bit error rate of $2.2 \times 10^{-4}$ at a data rate of $16000 \mathrm{bps}$ was achieved using a micro-pixel light emitting diode array and 4-ary pulse amplitude modulation.
\end{abstract}

Keywords—optical camera communications, discrete pulse amplitude modulation, LED array.

\section{INTRODUCTION}

Optical camera communications (OCC) is an emerging technology, enabling communication using off-the-shelf cameras and light emitting diode (LED)-based transmitters (Tx's). Cameras can offer multiple functions such as vision, positioning, and communication, simultaneously. However, the data rate in OCC is limited normally to the frame rate of the camera. Different techniques including multi-input multi-output (MIMO) [1], colour shift keying [1], and equalization [2] have been employed to enhance the data rate in OCC. Moreover, complex modulations such as $m$-ary quadrature amplitude and phase ( $m$ QAM) [3] and $m$ ary pulse amplitude modulation $(m \mathrm{PAM})[4]$ can boost the data rate to $\log _{2} m$ times the frame rate of the camera. However, complex modulation schemes require a digital to analog converter (DAC) to generate the electrical signal. Complementary metal oxide semiconductor (CMOS) image sensors with the implemented rolling shutter (RS) exposure mechanism can map temporal variation in the intensity of light into spatial changes in the image. This feature can be used to significantly improve the data rate in OCC by hundreds of times the data rate of the camera [4]. In the case of $m$ PAM, the variations in the light intensity in the RS mode forms multiple bands with different brightness, see Fig. 1(a). In the absence of a DAC, an LED array can be employed to generate an analog signal by aggregating the intensity of individual LEDs, also referred to as digital-to-light conversion (DLC) [5]. In this paper, we demonstrate a DLC technique for PAM similar to [5] for non-line-of-sight RS-based (NLOS) OCC.

\section{SYSTEM MODEL}

Figure 1(a) illustrates the schematic system model used for this work. A $\mu$ LED array with a size of $M \times N$ transmits the optical information and a lens system projects the light onto a wall/screen in defocused mode. At the receiver side, an RS camera with a resolution of $U \times V$ captures the variations in the intensity of the reflected light, i.e., a $(M \times N) \times V$ non-imaging MIMO link at each time instant. Every symbol of the data generated in $m$ PAM modulation is mapped to binary forming $\log _{2}(m)$ bits/symbol. Each bit is sent to a separate column of the LED array corresponding to its bit value. On the LED array, all LEDs within one column transmit the same data but independent of other columns (labelled c1, c2, and c3 in Fig. 1(b)). Note, the sum intensities (SIs) of transmitting columns are different corresponding to the bit value and a power of 2 times the column with lowest SI. That is, the column with lowest SI transmits the least significant bit, while the most significant bit is transmitted by the columns with the SI of $m / 2$ times the lowest SI. As a result, a $m$-level illumination pattern is formed on the wall by aggregating the illumination of different columns, representing the same symbol of the generated PAM signal. Note, it is essential that Tx optics create a highly defocused footprint on the wall/screen so that the footprint of all LEDs fully overlap. Accordingly, at each time-step, labelled $\mathrm{t} 1$, $\mathrm{t} 2$, and $\mathrm{t} 3$ in Fig. 1(b), a striped pattern with discrete multi-level illumination is captured, see Fig. 1(c). The images taken are next processed to extract the data. In order to improve the signal to noise ratio (SNR), we element-wise average the values of all columns. However, this imposes distortion to the signal due to the shape of the footprint and illumination pattern. To compensate for the distortion, a picture of the footprint with no AC signal is taken to obtain a calibration curve. Next, we element-wise divide the received signal by the calibration curve; see Fig. 1(d). The SNR at the edges of the footprint is very low and dividing by a small number imposes a high noise level. Therefore, we truncate the signal on both sides to ensure a reasonable SNR for the received signal. Note, this method can also be implemented in line-of-sight OCC, provided that a large defocused footprint of the light source can be created on the image sensor.

\section{RESULTS}

We evaluated the proposed concept experimentally. The system setup comprised a $16 \times 16$ flip-chip bonded array of GaN $\mu$ LEDs with $100-\mu \mathrm{m}$ pitch, $72-\mu \mathrm{m}$ pixel diameter, a bandwidth of $110 \mathrm{MHz}$, and a peak intensity at $410 \mathrm{~nm}$; a camera lens with a FoV of $\sim 4.5$ degree; a white wall; and an iPhone-7-plus wide-angle rear camera. The camera, the LED array and the optics were positioned at a height of $25 \mathrm{~cm}$ from the base, all facing towards the wall, creating an NLOS link. The ISO and exposure time of the camera was 
set to 1760 and $125 \mu \mathrm{s}$, respectively, and the resolution of the camera was $3024 \times 4032$. To obtain the calibration curve, we took 20 pictures of the footprint with no AC signal on and took an average over the pictures and the columns. This ensures a reduced temporal noise standard deviation, hence a low noise calibration curve with a size of $3024 \times 1$. We generated 4000 bits in 4 -PAM format and passed it to the system in 10-symbol long packets and captured $1000 \mathrm{JPG}$ images of the footprint. For each image, an average over all columns was taken, leaving an array with a size of $3024 \times 1$. This array is then elementwise divided by the calibration curve. Figure 2 (a)-(c) show the eye diagram of the 4-level received signal for $R_{\mathrm{s}}$ of 2000,4000 , and $8000 \mathrm{symbol} / \mathrm{s}$, respectively. We sample at the maximum eye opening to minimise the impact of inter-symbol interference (ISI). We measured the mean and standard deviation of each level and estimated the bit error rate as $9.5 \times 10^{-8}, 9.2 \times 10^{-8}$, and $2.2 \times 10^{-4}$, respectively, which are all below the $7 \%$ forward error correction limit of $3.8 \times 10^{-3}$. Therefore, we achieved a data rate of $16 \mathrm{kbps}$. Note, the eye closes as the $R_{\mathrm{b}}$ increases. This is due to the ISI imposed by the exposure time, i.e., to open the eye a shorter exposure time must be used at a cost of reduced SNR.

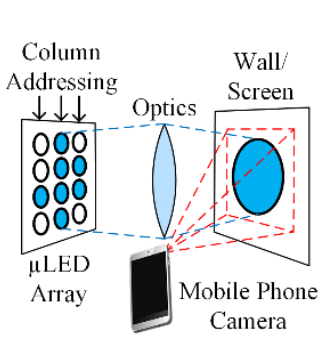

(a)

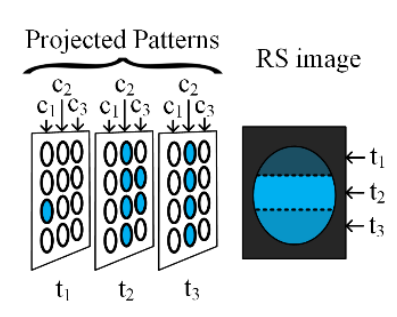

(b)

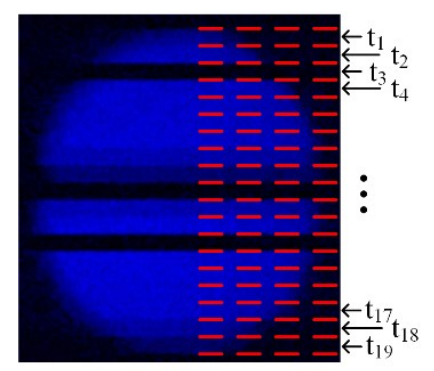

(c)

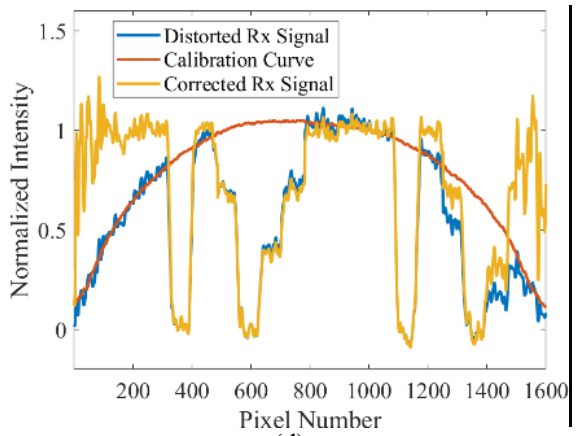

(d)

Fig. 1. (a) Schematic of the setup, (b) illustration of discrete PAM encoding and received image with RS effect, (c) experimental result, where the horizontal red lines indicate symbol boundaries, (d) Normalized intensity as a function of pixel number for the received signal.

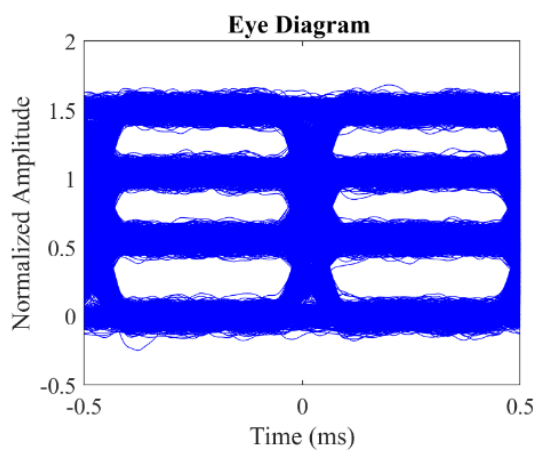

(a)

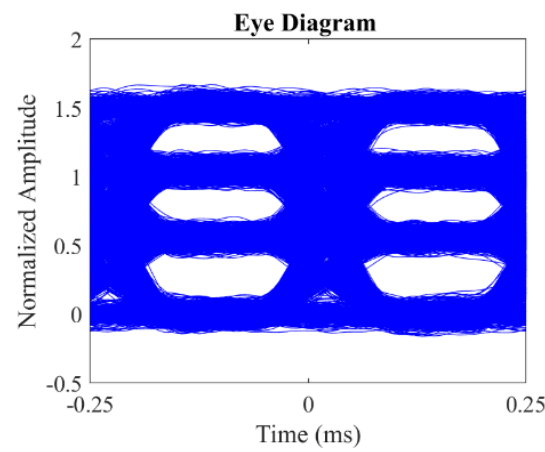

(b)

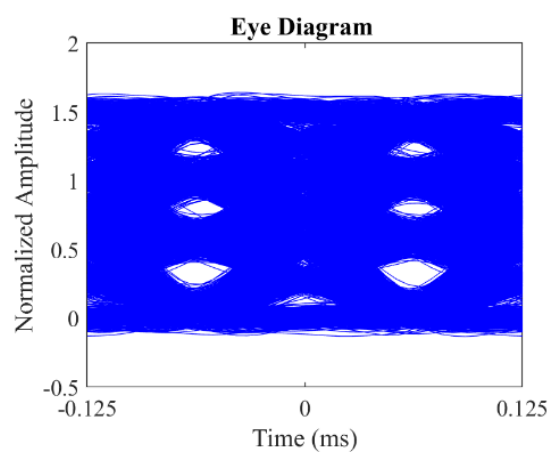

(c)

Fig. 2. Eye diagram of the received signal for $R_{\mathrm{b}}$ of (a) $2 \mathrm{ksymbol} / \mathrm{s}$, (b) $4 \mathrm{ksymbol} / \mathrm{s}$, and (c) $8 \mathrm{ksymbol} / \mathrm{s}$.

\section{CONCLUSION}

In this paper, we demonstrated experimentally that the digital-to-light approach is readily implemented in optical camera communications and helps to increase the achieved data rate; in our case by a factor of 2 compared with conventional RS-OCC using OOK modulation schemes.

\section{ACKNOWLEDGMENT}

This work was funded by EPSRC grant EP/S001751/1 and data are available at https://doi.org/10.15129/de7b702f-aa64-47529756-d24f96c19d58.

\section{REFERENCES}

[1] W. Huang, P. Tian, and Z. Xu, "Design and implementation of a real-time CIM-MIMO optical camera communication system," Optics express, vol. 24, pp. 24567-24579, 2016.

[2] O. I. Younus, N. B. Hassan, Z. Ghassemlooy, P. A. Haigh, S. Zvanovec, L. N. Alves, et al., "Data Rate Enhancement in Optical Camera Communications using an Artificial Neural Network Equaliser," IEEE Access, 2020.

[3] T. Nguyen, M. D. Thieu, and Y. M. Jang, "2D-OFDM for optical camera communication: Principle and implementation," IEEE access, vol. 7, pp. 29405$29424,2019$.

[4] V. P. Rachim and W.-Y. Chung, "Multilevel intensity-modulation for rolling shutter-based optical camera communication," IEEE Photonics Technology Letters, vol. 30, pp. 903-906, 2018.

[5] A. D. Griffiths, M. S. Islim, J. Herrnsdorf, J. J. McKendry, R. Henderson, H. Haas, et al., "CMOS-integrated GaN LED array for discrete power level stepping in visible light communications," Optics express, vol. 25, pp. A338-A345, 2017. 